\title{
Teleeducation and telepathology for open and distance education
}

\author{
Janusz Szymas \\ Department of Clinical Pathology, Karol \\ Marcinkowski University of Medical Sciences, \\ Przybyszewski Str. 49, 60-355 Poznan, Poland \\ Tel.: +48618691498; \\ E-mail: jszymas@ampat.amu.edu.pl.
}

Our experience in creating and using telepathology system and multimedia database for education is described. This program packet currently works in the Department of Pathology of University Medical School in Poznan. It is used for self-education, tests, services and for the examinations in pathology, i.e., for dental students and for medical students in terms of self-education and individual examination services. The system is implemented on microcomputers compatible with IBM PC and works in the network system Netware 5.1. Some modules are available through the Internet. The program packet described here accomplishes the TELEMIC system for telepathology, ASSISTANT, which is the administrator for the databases, and EXAMINATOR, which is the executive program. The realization of multi-user module allows students to work on several working areas, on random be chosen different sets of problems contemporary. The possibility to work in the exercise mode will image files and questions is an attractive way for self-education. The standard format of the notation files enables to elaborate the results by commercial statistic packets in order to estimate the scale of answers and to find correlation between the obtained results. The method of multi-criterion grading excludes unlimited mutual compensation of the criteria, differentiates the importance of particular courses and introduces the quality criteria. The packet is part of the integrated management information system of the department of pathology. Applications for other telepathological systems are presented.

Keywords: Telepathology, teleeducation, automated score, teleteaching

\section{Introduction}

Teleeducation is the use of multimedia communication components in teaching, education, and examination at a distance. The most important factors, which have influenced the distance education, were broadband telecommunication links and development of electronic multimedia techniques [5,10,14,15].

The speed of exploring new technical, biological or social information is increasingly combined with the task of shortening the gap between theoretical exploration and practical application. The period of transferring the collected information from specialized centers to interested students has to be shortened, and those countries that can provide their students with the latest science in the shortest time are in an advantage. In addition, the absolute and relative number of students is increasing. The more detailed the education, the more specialized teachers are needed, and, consecutively, the more expensive is the education. Teleeducation is an appropriate technical solution fulfilling the needs of economy and essential application $[2,11,14$, 17]. Education is closely associated with results in scientific research. The volume of medical information is constantly growing, being estimated to double every 5 years. As a consequence, an increase in the demand for rapid access to the latest results of scientific research, new therapies, and effects of new medicines to be implemented in related education systems is observed. To solve this problem on a continuous basis, it is appropriate to develop a system of central medical information that would be accessible to students, trainees, or doctors at any time. Such a system should offer access to various sources of information by means of electronic mail or on-line communication $[1,6,7,13]$.

There is the conviction that expanded network systems in medicine will become, apart from the industry or service sector, the main force enhancing the development of digital and broadband telecommunications. Research results of these institutions must be available for medical education immediately. The natural application of teleeducation is the use of videoconferences for transmitting a surgery course or for remote access to multimedia databases. In addition, medical professionals require continuous education and training to maintain and improve their skills. Application of teleconferencing can accomplish official training programs from specialized medical centers located 
in large cities to local hospitals, private institutes and homes. It could be used in training of specialized medical staff: physicians, nurses and therapists. Patient's relatives can be trained by observation and interactive guidance to care for the patient at home or in small care units. The appearance of new diseases and outbreaks of epidemics following natural disasters require immediate access to information of treatment and methods for adequate examination and prophylaxis. New technologies allow the transfer of knowledge from the most accomplished scientific personalities and lecturers to persons who have never entered a lecture room $[2,10]$. Without any technical difficulty it is possible to organize telelectures with interactive telecommunication between the teachers and students who could attend the presentation at home. These offerings will alter the world of education and training by specific distributed networks and digitized interactive television sessions.

\section{Telepathology}

The development of computer science and dissemination of microcomputers permit the use of the techniques of electronic data interchange in all departments of pathology. Production and communication of digital images is now daily used among pathologist involved in histopathological diagnosis, for staff meeting, for continuous education or for second opinion [7, 10]. Due to decrease in price of the imaging systems, new range of users will have the opportunity to share image information locally, abroad or global. Staticimaging and dynamic-imaging are the two major competing technologies of telepathology [10].

Telepathology is the diagnostic work of a pathologist at a distance using telecommunication links [10]. It includes specific application fields, which require specific technical solutions. These include frozen section service, expert consultations, remote control measurements, and education and training. Applications effect, all aspects of diagnosis, especially those involved in the daily workflow of a pathologist [7]. The image quality, transfer rates, and screen resolution of telepathology systems are sufficient for an additional or primary judgment of histological slides and cytological smears. It is, therefore, possible to include this technique into inter-laboratory quality control of all steps of diagnostic procedures and to use this technique for quality assurance and control [7]. Telepathology is, therefore, not a substitute of conventional diagnos- tic procedures but a real improvement in the world of pathology [6-10].

It is of importance to consider the fact that pathology is a unique scientific specialty, which requires complex and careful testing, comparative studies, and ethic control in new diagnostic strategies. Moreover, there is a need for a rapid exchange of information within the framework of cooperation between various clinics of a particular specialty or specific units of pathology such as neuropathology, dermatopathology, lung pathology, etc.

The main relevance of telepathology lies in its capability to link electronically pathologists in remote hospitals to larger or specialized pathology institutes. This may become even more important due to current increase in sub-specialization and the demand for more precise diagnosis and consultation in difficult cases [10]. A network connecting small clinics or laboratories to larger and more specialized units, and to highly specialized reference centers may improve the professional standard of health care services and education. For a wider use, a technological standardization will be required, since the existence of several types of computers and numerous image manipulation programs have resulted in a proliferation of file formats. However, every potential user or client of telepathology should keep in mind, that standardization also includes legal and ethic issues such as patient confidentiality or potential malpractice [10]. An adaptation of work guidelines and protocols is required. For the transmission of the digitized images from a telemicroscope to the remote diagnostic video monitor, different technologies such as ordinary telephone lines, broadband telecommunications channels, or the Internet can be used. The transmitted images may serve for primary pathological diagnosis, teleconsultation, quality assurance, proficiency tests, or distance learning. Some countries have introduced and consequently fund projects for telepathology for education in pathology as a project of relevant national interest. For example, such a project is headed by the Institute of Pathology at the University of Udine, and currently involves additional four Italian universities (Ancona, Bari, Ferrara, Sassari). Such activity will allow collaboration among participating pathological departments and will also boost other medical disciplines, becoming in this way a model which can be adopted for problem-based medical education [1]. 


\section{The Internet}

The installed network is based on standard protocols and communication tools, with particular attention to the Internet. Both main sets of its protocols, i.e., e-mail and WWW, allow the pathologist easy access to network resources. The main network activities are: development of collaborative research projects based on telematic tools; creation of multimedia case archives for education and continuous education; collaborative authoring of multimedia educational material; quantitative pathology studies, study and application of telematic methods for quality control in surgical anatomic pathology. The Internet allows a direct interaction and real-time discussion between the student and the professor at any distance. Increasingly, nearly every field of human knowledge and information is available on the Internet, often presented in an attractive manner. Telecommunication in science will prepare the field of combined research and might bridge the increasing gaps between the scientists working at the bases in the different specialized research areas such as molecular biology. Relatively inexpensive tools may allow pathologist to increase collaboration, not only in the now traditional telepathology fields such as telediagnosis and remote consultation, but also for educational and quality control aims. Furthermore, every form of collaboration has a secondary effect on continuous education of the students and pathologists.

\section{Telepathology as a tool for medical education}

\subsection{Practical course of Gross Pathology on Web server}

The information service on Web server can be used for the course of gross pathology with weekly classes during the semester curriculum. Standard technologies like HTML and JavaScript may be used for the information service. As soon as the results of an autopsy were available, a short clinical description of the case was presented on the pathology's web site (http:// www.mi.med.uni-goettingen.de/PathologieLehre/) together with a small number of questions guiding the students through the case [6]. These questions were discussed in the subsequent class. The evaluation was carried out with a semistructured questionnaire. The answers were subsequently classified and analyzed by descriptive statistics. The study was performed to determine the feasibility of a comparative, objective as- sessment of the learning outcome in users and nonusers of the service. With a relatively simple, straightforward technological approach, a favorable response of the students has been achieved. A majority of the students uses the information service on a regular basis and considers it a useful information source. The technologic and organizational design of the service permits a flexible improvement of contents on the basis of the survey results and of the teachers' experiences.

\subsection{Virtual slide as teaching tool}

A virtual slide is a fully digital representation of the histological specimen by means of a series of static color images $(800 \times 600$ pixels, 24-bit) taken at low $(5 \times)$, medium $(10 \times, 20 \times)$ and high power objective $(40 \times, 63 \times)$. The implemented architecture of the system is constituted of two modules. The first module allows the acquisition of images by an automated capture of image of the entire specimen. All images are storage on magnetic or optical devices. In combination with clinical and radiological data a construction of the virtual case is possible (Fig. 1). The second module permits the examination of the virtual case, and consists of a user-friendly interface. The pathologist can select and visualize all images of interest. The software modules are written in Java. The users interface is based on the TELEMIC system [16]. By viewing a virtual slide, the students can screen the slide at low-magnification, and examine areas of interest at (Fig. 2) higher magnification. The student examines the slide similar to a consultant pathologist and renders a remote diagnosis by examining the slide through a robotic microscope. The aim of this tool is to provide the students with case-based information for the practical experiences in pathology and the next higher class is specifically prepared for these causes. The students perform the presentation, and the teacher can more effectively focus an differential diagnoses and the diagnostic strategy.

\subsection{Continuous education}

Continuous education in pathology is possibly by and of the international servers dedicated to cooperative work and to publish images (Fig. 3) and clinical reference databases by a telepathology network. The first step, the cooperation was achieved by definition of communication standards as a set of univocally identified high quality JPEG images, a coded data sheet and a clinical document. For this new tools for accessing, communicating and displaying images 


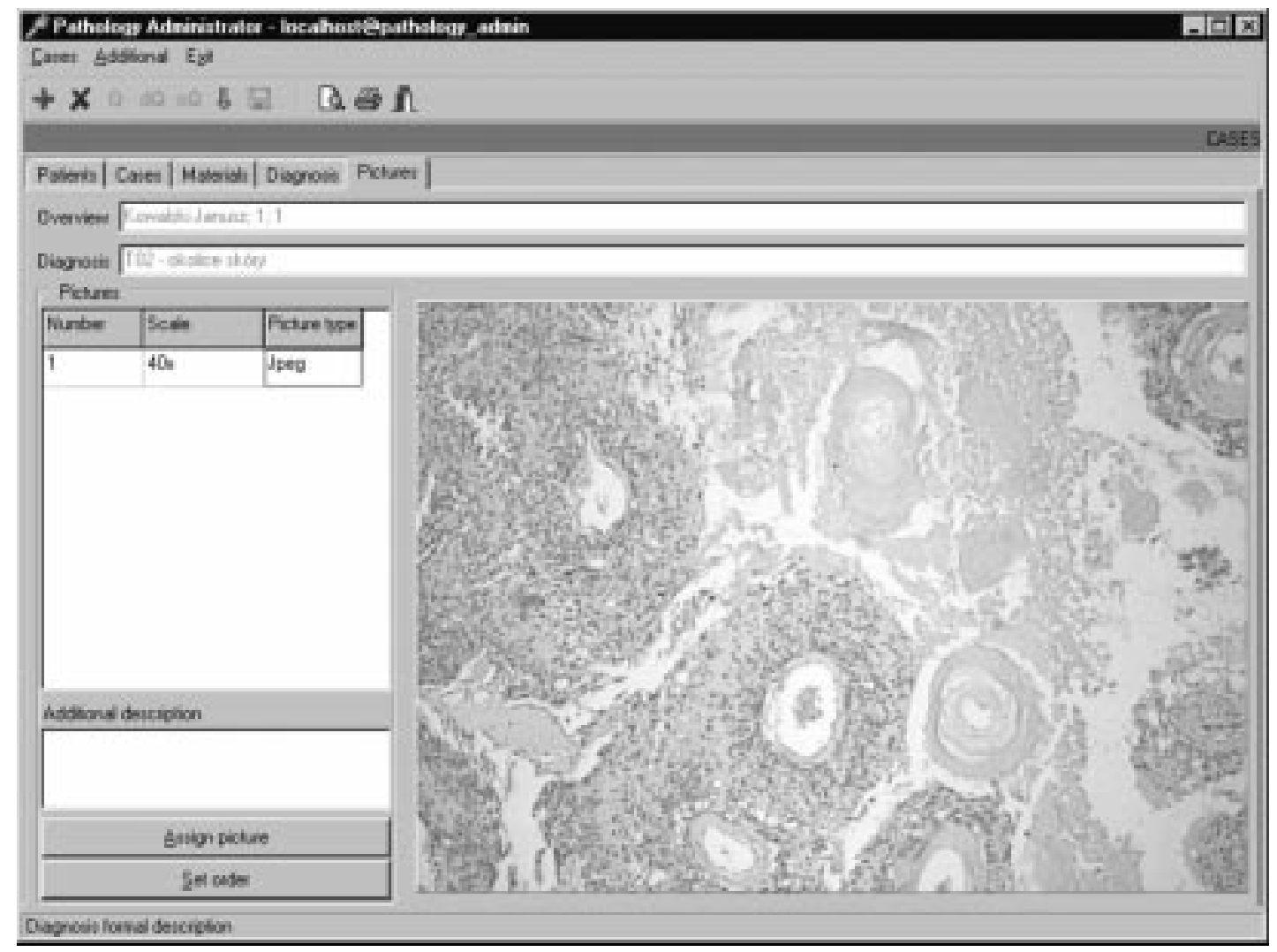

Fig. 1. Screen of data base of text and image files for the construction of virtual case. Management system is based on MySQL, HTTP and PHP languages.

associated with clinical data activity also developed. The intercommunication server allows the exchange of standardized folders through the Internet or ISDN. It also provides archiving facilities. Until now, the intercommunication server - ADICAP server at CRIHANROUEN - is used by 600 pathologists and hematologists through 200 workstations [2]. It allows the exchange of information for scientific publication and for second opinion service. Three series of publication has been launched: An atlas of pathology, distributed worldwide by the Springer-publishing company, Séminaires de Pathologie (mainly yearly French scientific communications distributed through scientific associations) and Tutorial Series. Editing is made by an scientific association or by the individual author. The published volume measures about 1000 folders of ten images per year.

\subsection{Multimedia database for self-education and testing}

A prime concern of evaluation is to monitor the students' experiences with the information service, en- abling its continuous and systematic improvement. For evaluation of students' knowledge most departments use test questions. The test questions have been used in our Department of Pathology for over 25 years to verify the students' knowledge. They had been collected in a preliminary note and issued some years ago. This edition enclosed 1412 questions [3]. However, it was not in a friendly form for teaching aid, for both the self-education of students and their self-determination. The elaboration included neither the right answer, nor the images. Therefore, the idea emerged to establish a system, managing the multimedia examination questions databases. The program packet was implemented on microcomputers compatible with IBM PC which works under the network system Netware 5.10 (Novell) for 50 users. The software is written in Clipper and Pascal languages. The packet consists of two functionally individual programs: ASSISTANT, which is the administrator for the databases, and EXAMINATOR, which is the executive program [4]. These two programs are implemented in the technique of pull-down 


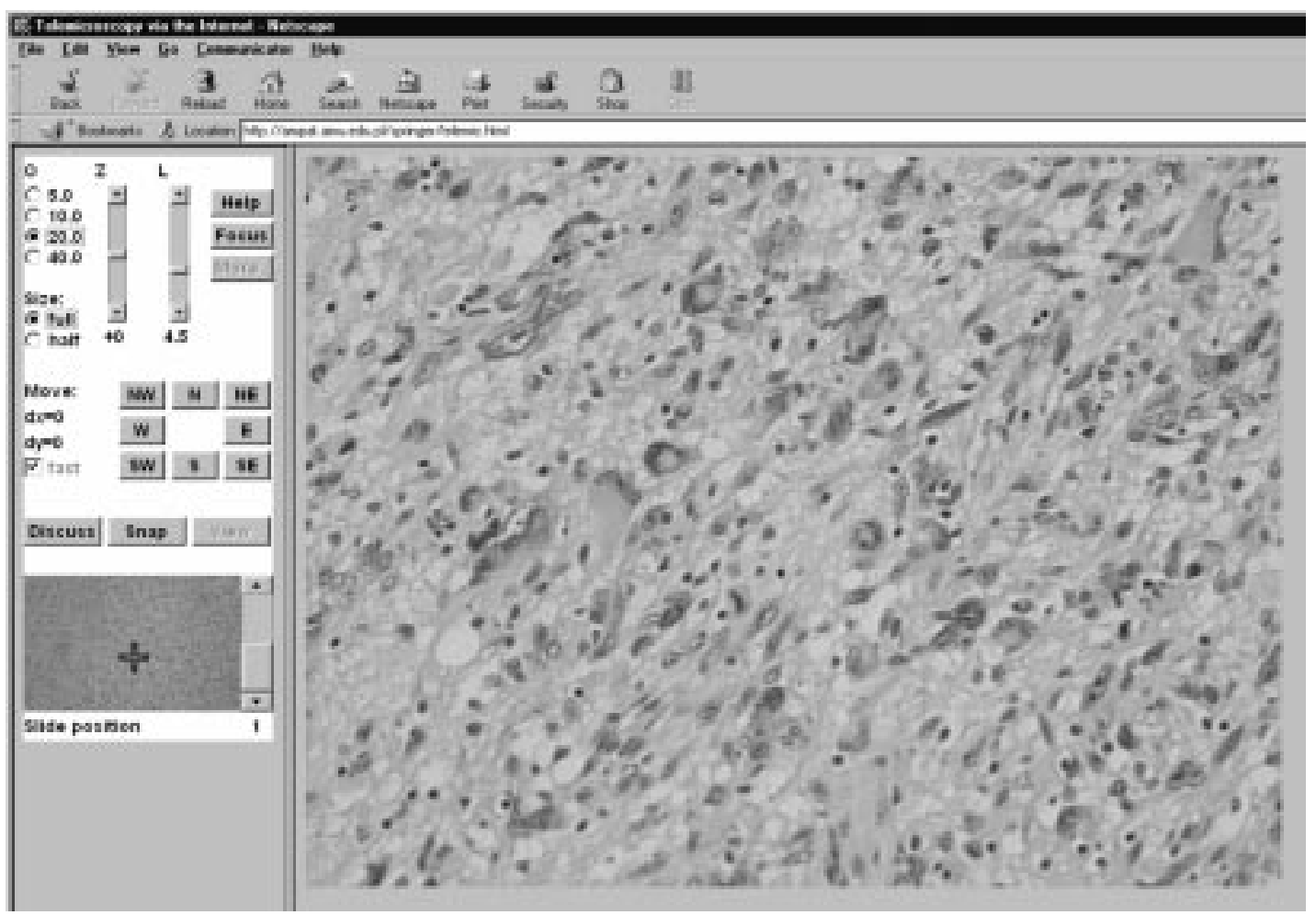

Fig. 2. User's interface of TELEMIC system for the display of virtual slide with visible area of interest at the higher magnification.

menu, message and selection windows. This way it is easier to be operated by inexperienced users.

\subsection{System of multi-criterion grading of students}

When classifying students according to the arithmetic mean of their scores served disadvantages have to be taken into account [12]. The new procedure divides students into categories related to the accepted grade scale. This procedure takes into consideration the importance of particular sections and the influence of the assistant in charge of particular course compartments. We suggest the division of students into five categories, each consisting of two subcategories. In addition, this procedure qualifies some students beside the multiple-choice exam for an oral exam inactive in order to give them a better grade. Exemplary application of this method was tested on a group of 358 dental students previously graded by "the method of mean" in pathology. The method of multi-criterion grading excludes unlimited mutual compensation of the criteria, differentiates the importance of particular courses, and introduces a quality criterion that was not used before, i.e., the category of person conducting the course section. It allows to define the group of "questionable" students who should take an additional oral examination in order to be graded properly. Creating and using the system of multi-criterion grading of students in the pathology course is an improvement and given a more objective and flexible time [12].

\section{Examples of the program use}

This program packet is currently working in the Department of Pathology of University Medical School in Poznan. It is used for self-education, tests, services and the examination in pathology, for all dental students in the Medical Department and for self-education and individual examination services for medical students. The results together with the notes obtained for their activities in practical classes create the basis to credit particular sections and the final examination. The implementation of this packet with test question set helps to standardize the teaching results in pathology in Poland. This packet is part of the integrated management information system of the pathology department [13]. 


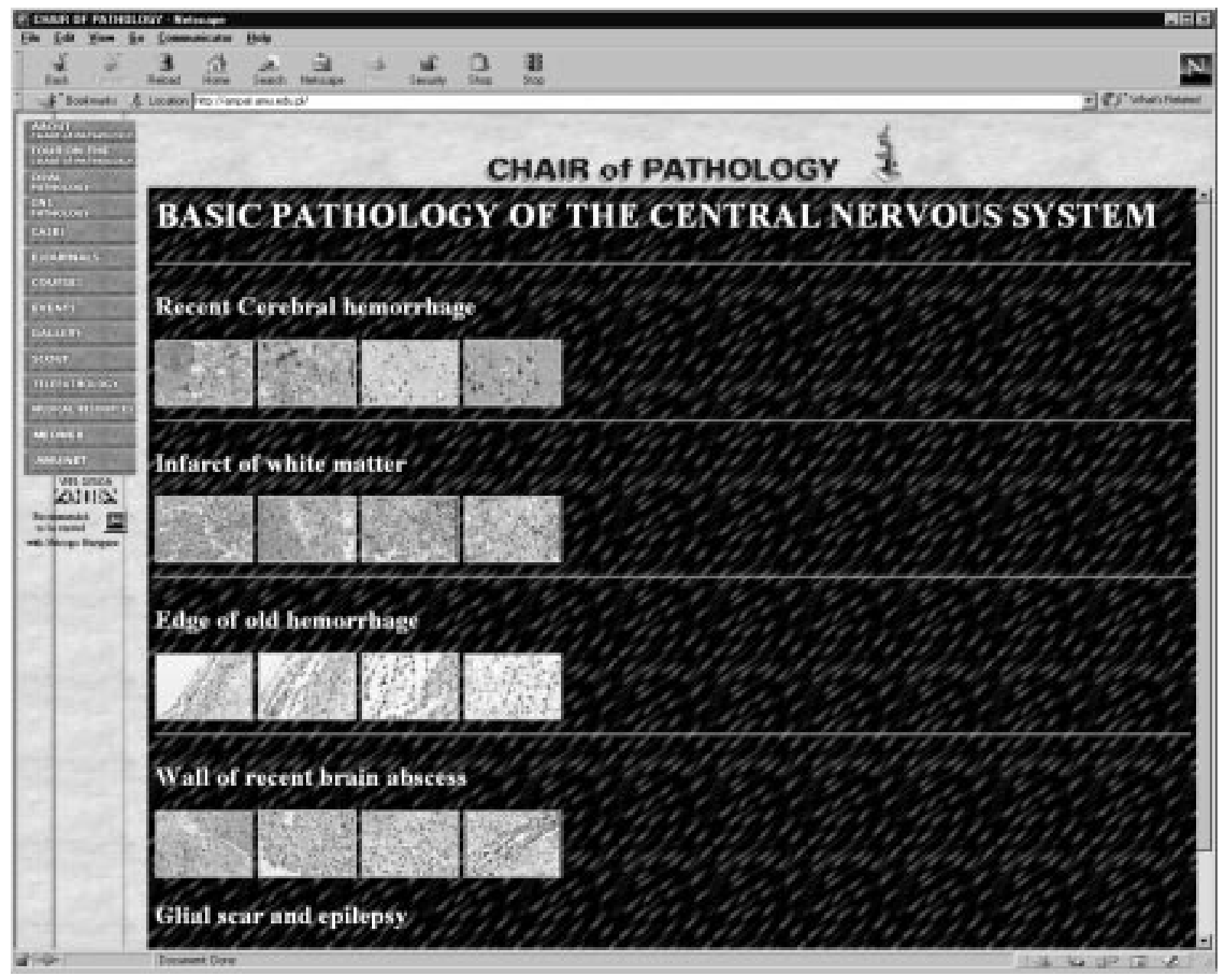

Fig. 3. Gallery of microscopic pictures of basic pathology of the central nervous system at the WWW server.

\subsection{ASSISTANT}

The ASSISTANT program creates and defines any database [4]. It enables the import of text files and images, which will be displayed on request. The images are stored in separate files. The program has no restriction concerning the image file format because it uses the external-display program. It is also an instrument to understand the database administration, which includes the questions. Regarding the possibility of many thematically different databases, it enables to create a separate configuration for each of them, which is called the project session. This project includes answer mode, the time of answering, number of the question, method of question selection from the base, and masking them. Except these functions, the ASSISTANT program enables to review the reports of the sessions that have been hold before and also to prepare the data for statistical analysis.

\subsection{EXAMINATOR}

The EXAMINATOR is an executive program created for self-education or examination of students. The configured file created by the ASSISTANT program is the base of this program operation. This file is invisible to the user. After calling the executive program it starts to work according to the session project which had been created before. The program ask for the name of the student and informs about numerical buttons which may be used by the examined person in order to answer the questions. The number of the answer buttons must be in accordance with the number of available answers. If a picture is available, picture key is visible in the message line. After every answer, the system in- 
forms whether the answer is right and indicates the actual percent and numerical answer account of the examined person. The examination ends by display of the examination time and the results. This information is then stored (see also Figs 4-6).

\subsection{NOS}

NOS is software for the above-described system of multi-criterion grading of students [12]. It allows recording and updating personal data from students, their grades from particular course sections and from the final examination. It also allows to store data from previous years in the so-called archives. The main screen consists of three parts: the main menu, which contains basic options, the working part, and the bottom line with information about conducted operations. The main menu consists of six commands, i.e., add, find, delete, review or modify the data about assistants into running the courses. The Dentistry command can add, find, delete, review, and modify data of students. The data contain the following fields: credit book number, group, name and surname, assistant, year of studies, choice of exam dates (repeats), and the final grade. The Calculations command allows to perform: Classify and Results. The first one classifies all students from the group processed, the second one allows to search for certain categories.

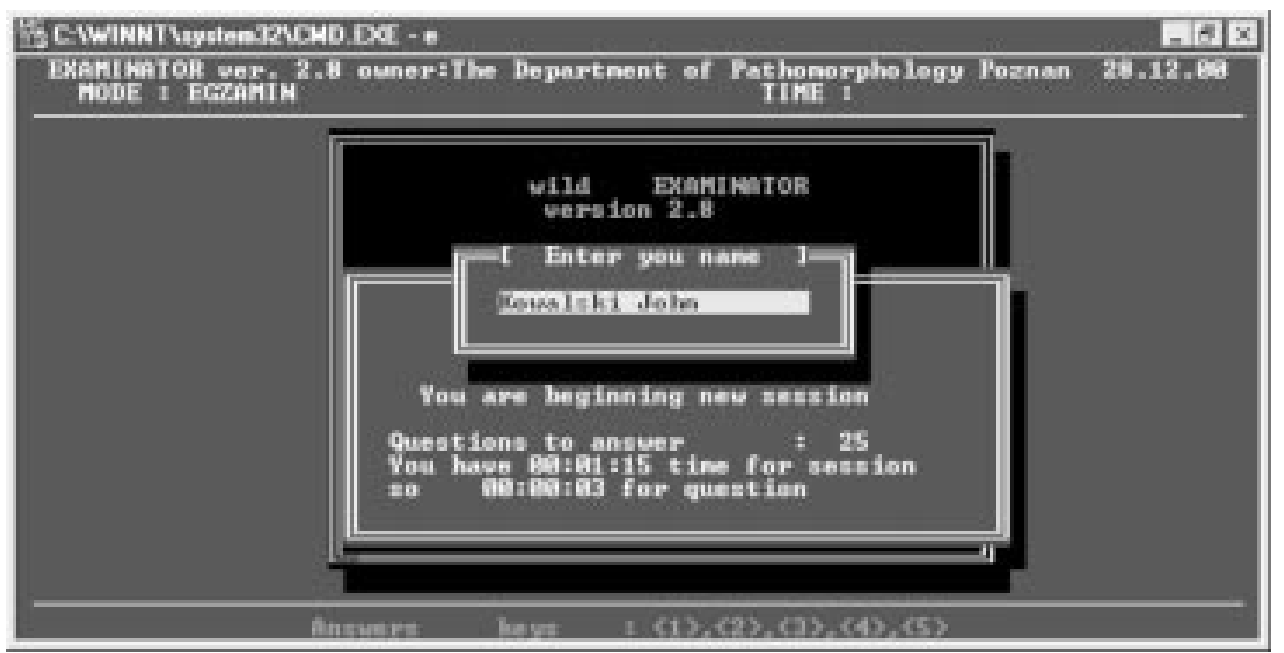

Fig. 4. Beginning of the exam with giving the family and first name. Below the information about conditions of an examination mode established by ASISTANT program is displayed.

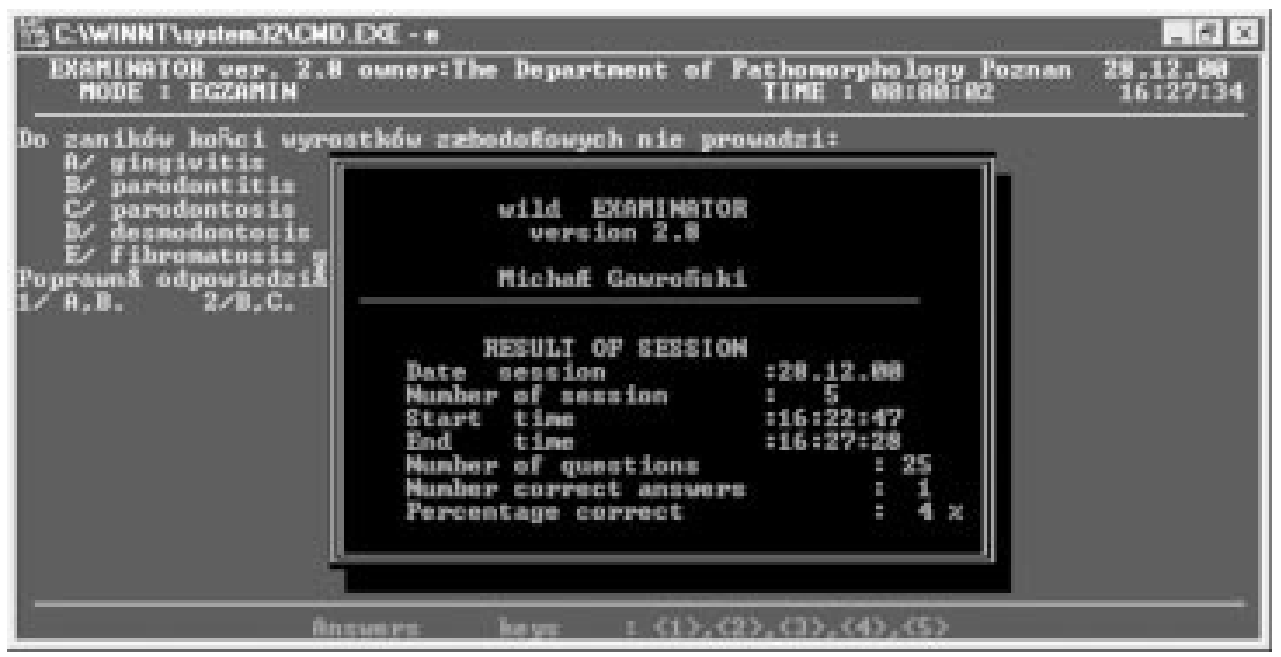

Fig. 5. Display of summary of final result at the examination end. 


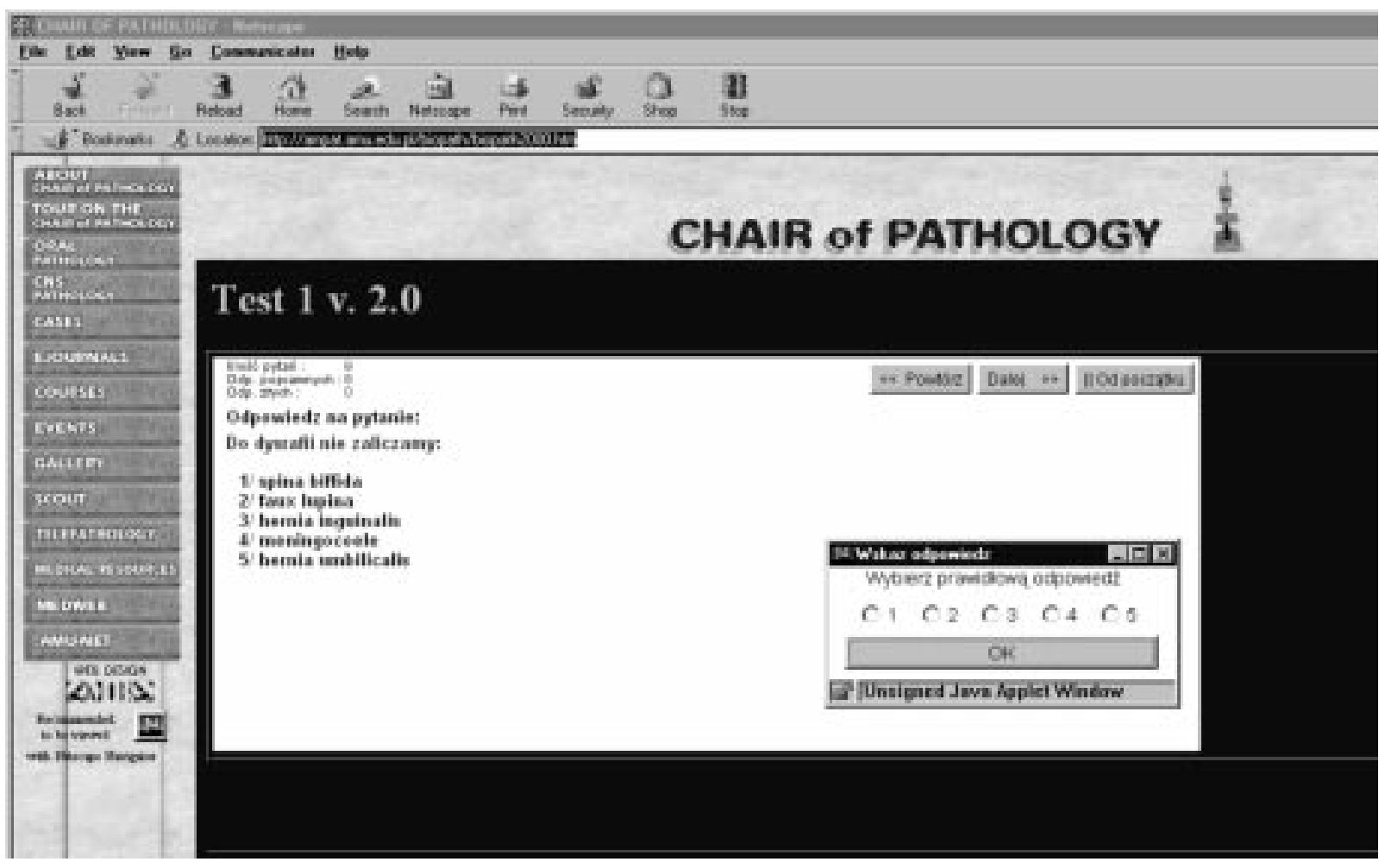

Fig. 6. Implementation of EXAMINATOR program on the WWW server for self-testing, available through the Internet.

NOS co-operates with the database about students, set up in STUDENT [15]. Classifying a student into a proper category is obtained by choosing the command and establishing the access paths to the database and the archives. After typing the credit book number, the monitor displays a window of dialogue with the student's data and suggested final grade. If the student is to take an additional oral examination, the grade will be accompanied by the comment: suggested. This grade can be changed later. The procedure also allows to store in a current catalogue only data about students who are not yet classified. Outranking coefficients can be displayed, which may appear helpful in case of doubts about the correctness of classification.

\subsection{TELEMIC}

This telepathology system is based on a fully automated microscope Axioplan2 that is directed by JAVA programs through a standard Internet browser. The remote microscope can be is assessed through a webpage on the website (http://mic.amu.edu.pl/). The major functions include the control of the stage and the control of microscope. The microscope and camera control software was integrated into an Internet server.
The server executes these commands, converts the captured images into compressed JPEG image files and distributes these image files to the all connected telemicroscopy clients. The number of students that can be connected to one microscope server is limited only by capacity of RAM memory. Each function can be controlled either by the mouse or the keyboard. The use of the Internet is not a necessity for the telemicroscopy software. The system is used in a local area network (LAN), which connects classrooms with the laboratory of the Pathological Department. Further features of the telemicroscopy system can be seen on the WWW page http://ampat.amu.edu.pl/.

\section{Perspectives}

The major gains in health in the 20th century were primarily the result of improvements in public health including sanitation and immunization. Global health improvements will occur in the 21 st century by improvement in information gathering and sharing. One way to improve health training and research is to improve the education. One of the most important aspects is the globalization of education. In the 
next century transnational training and global lectureshareware training will be available. Faculties will thus share their best, most passionate multimedia data on the Internet. Quality control systems and statistical quality assurance on the Internet will take place to monitor lectures given by experts all over the world.

\section{References}

[1] V. Della Mea, P. Cataldi, A. Marzola, L. Mariuzzi, G. Massarelli, R. Ricco, M. Scarpelli and C.A. Beltrami, The Italian Network of Telepathology: principles and first results. Abstracts. Vth European Congress on Telepathology, Elec. J. Pathol. Histol. 6(2) (2000), No. 002-12.

[2] J. Diebold, C. Got, J. Hémet and E. Martin, Continuing Education and Telepathology. Abstracts. Vth European Congress on Telepathology, Elec. J. Pathol. Histol. 6(2) (2000), No. 002-12.

[3] P. Gabryel, J. Szymas, W. Salwa-Zurawska and M. Gawronski, Test Questions on Pathomorphology. Part I. Examination Questions, Poznan, 1990 (in Polish).

[4] M. Gawronski and J. Szymas, The packet of teaching programs - ASSISTANT and EXAMINATOR version 2.5. Operating instruction, Poznan, 1992 (in Polish).

[5] K. Kayser and C. Kayser, Telepathology - aspects of social influence and quality control, Elec. J. Pathol. Histol. 3(3) (1996), No. 963-04.

[6] K. Kayser, G. Kayser and S. Zink, New technical aspects in telepathology, Elec. J. Pathol. Histol. 6(3) (2000), No. 003-04.

[7] K. Kayser and G. Kayser, Basic Aspects of and recent development of telepathology in Europe with specific emphasis on quality assurance, J. Anal. Quant. Cytol. Histol. 21 (1999), 319-328.
[8] K. Kayser, Telemedizin, Wiener Klin. Wschr. 108 (1996), 932940.

[9] K. Kayser, Telepathology in Europe. Its practical use, Arch. Anat. Cytol. Pathol. 43 (1995), 196-199.

[10] K. Kayser, J. Szymas and R. Weinstein, Telepathology. Telecommunication, Electronic Education and Publication in Pathology, Springer-Verlag, 1999.

[11] F. Leiner and L. Füzesi, Internet-based Support in Medical Education: Practical Course of Gross Pathology. Abstracts. Vth European Congress on Telepathology, Elec. J. Pathol. Histol. 6(2) (2000), No. 002-12.

[12] R. Slowinski, B. Wolynska, M. Kaczalski and J. Szymas, The method of multi-criterion grading of students in course of pathomorphology. Second National Conference "Computers in Medicine”, 1994, Lodz, pp. 101-106 (in Polish).

[13] J. Szymas and R. Dubiel, The integrated management information system of pathomorphology department, in: IX National Conference of Biocybernetics and Biometrical Engineering. Conference Materials, Vol. II, Gdansk, 1992, pp. 315-318 (in Polish).

[14] J. Szymas and M. Gawronski, Multimedia data base and management system for self-education and testing the students' knowledge on pathomorphology, Pol. J. Pathol. 44 (1993), 183-188.

[15] J. Szymas and J. Jelonek, STUDENT - Computer management system for didactic in department of pathology, in: Second National Conference "Computers in Medicine”, Lodz, 1994, pp. 111-116 (in Polish).

[16] J. Szymas and G. Wolf, Real-time microscopy through the Internet, Elec. J. Pathol. Histol. 4 (1998), No. 983-07.

[17] B. Wolynska, M. Kaczalski and J. Szymas, Computerized evaluation of students' knowledge in a course of pathology, Elec. J. Pathol. Histol. 6(2) (2000), No. 002-05. 


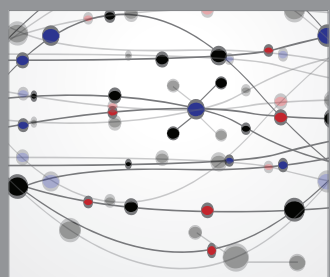

The Scientific World Journal
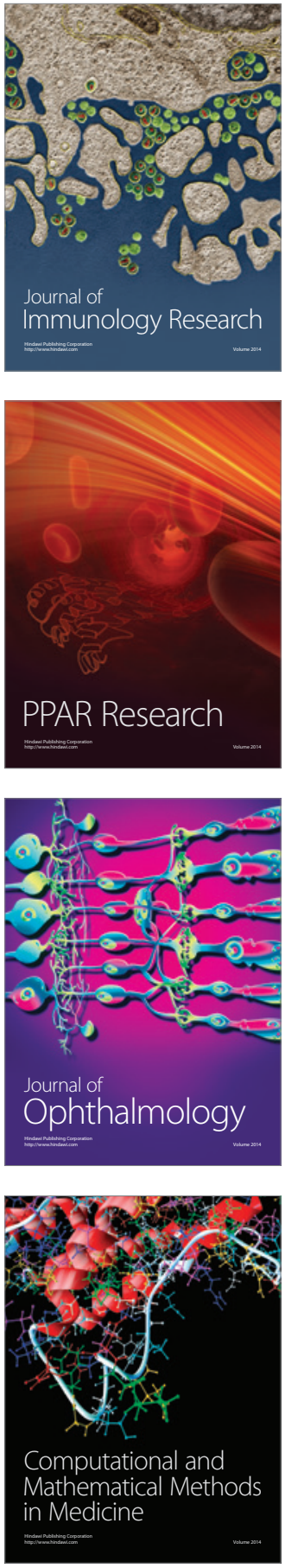

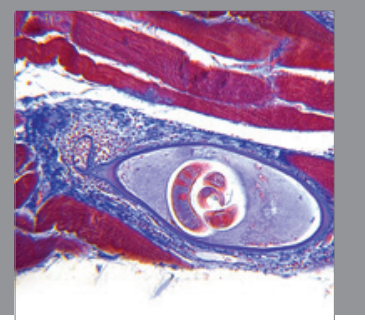

Gastroenterology

Research and Practice
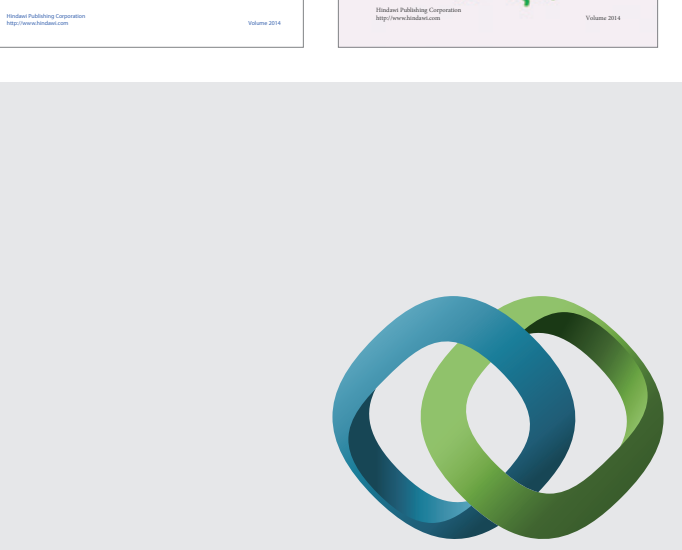

\section{Hindawi}

Submit your manuscripts at

http://www.hindawi.com
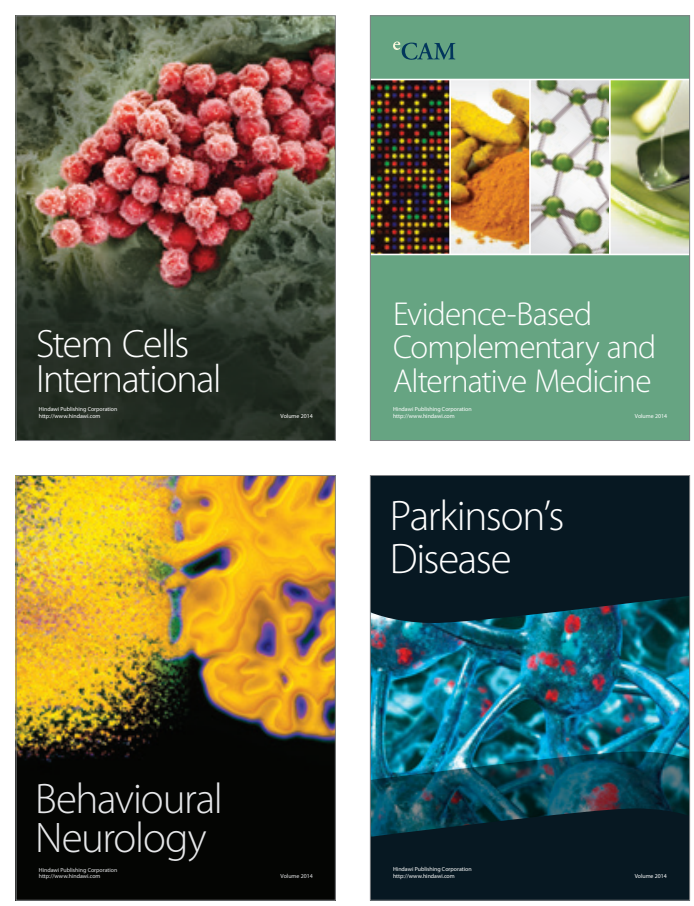

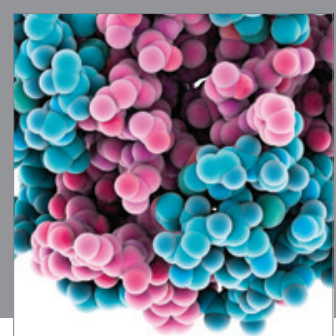

Journal of
Diabetes Research

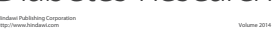

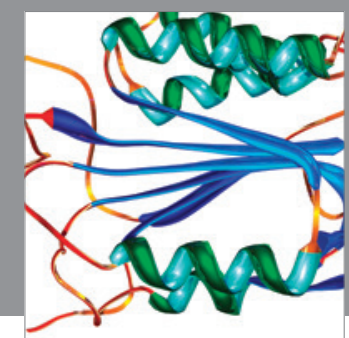

Disease Markers
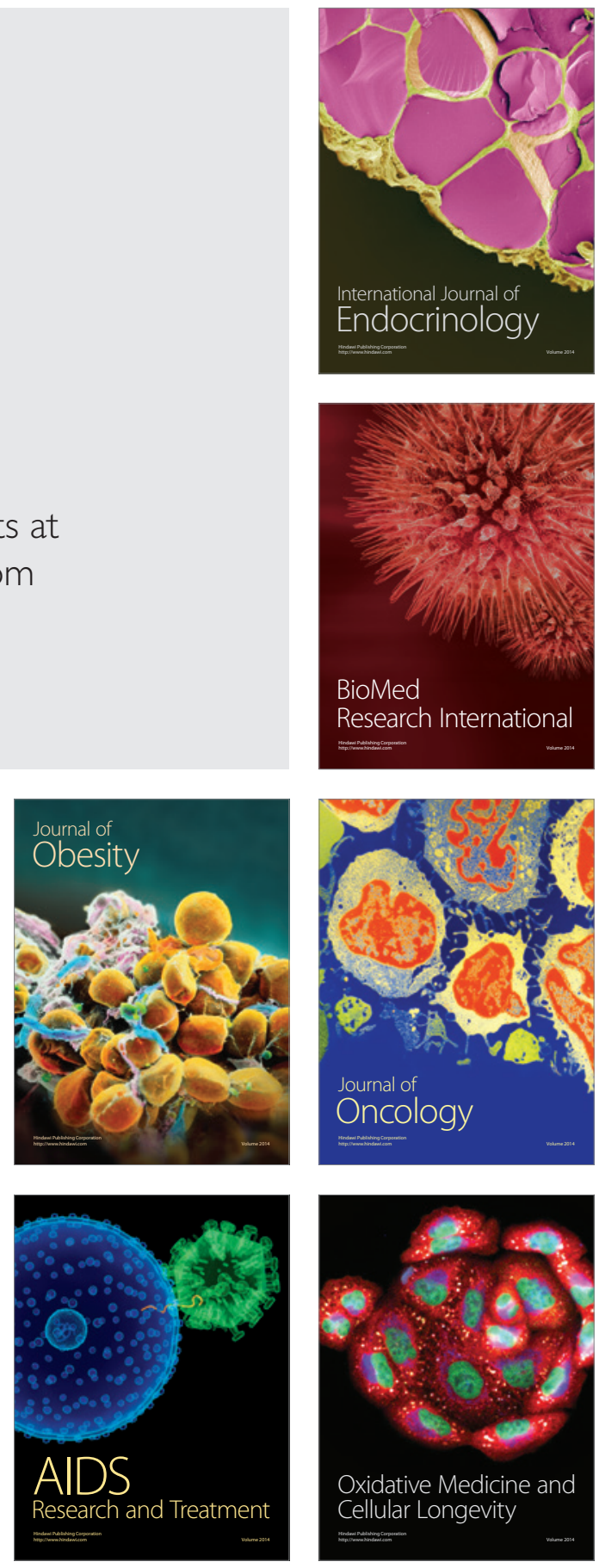